\title{
A STUDY OF THE CIRCLE CROSS*
}

BY

\section{J. L. COOLIDGE}

One fundamental characteristic of that discipline which is called by the vague title of "higher geometry" consists in taking as element, not necessarily a point in two or three dimensional space, but any object which can be determined by a finite number of parameters. The student of analytic geometry begins in Cartesian fashion with a point and its coördinates. A little later line and plane coördinates are introduced, and the student feels that the content of his subject has been multiplied by two. Further extensions soon follow, the Plücker line geometry, the coördinates of a circle or sphere, the linear line-complex as space element, the projective geometry of $n$ dimensions. The process is capable of indefinite extension.

In line geometry a very fruitful idea was introduced by Study, $\dagger$ that of taking as element, not a single line, but a pair of lines called a "cross." One line of the pair is finite, the other, in general, at infinity, and each plane through one is perpendicular to the other. The cross takes a more symmetrical shape in non-euclidean space where under the previous definition the two lines are merely mutually polar with regard to the absolute quadric. $\ddagger$ It is the object of the present paper to extend this concept to circles. A circle cross will be composed of a pair of circles so related that every sphere through one is orthogonal to the other. In the first section we shall give a number of preliminary theorems, some well known, concerning the angles of circles. In the second we take systems of circles orthogonal to one sphere. The analogy to the corresponding problem in non-euclidean line geometry is here so close as to be almost identity, and we shall give our results with all possible brevity. In section three we shall discuss circle crosses in general position in space. The simple analytical treatment of the cross as element does not seem possible in this case, but a number of interesting results are obtainable. In the last section we discuss a new transformation which carries circles into circles, but not spheres into spheres. Theorems already familiar are marked with an accent to the number, as Theorem $2^{\prime}$, etc. Others not so marked are believed to be new.

- Presented to the Society December 31, 1912.

† Geometrie der Dynamen, Leipzig, 1903.

$\ddagger$ See, e. g., the author's Non-Euclidean Geometry, Oxford, 1909.

Trans. Am. Math. Boc. 10 


\section{$\S 1 . \quad$ The Angles of Circles.}

We shall take as our domain what may be called a perfect pentaspherical continuum, i. e., a set of objects called points in one-to-one correspondence with all sets of values of five non-simultaneously vanishing coördinates *

$$
x_{0}: x_{1}: x_{2}: x_{3}: x_{4}
$$

connected by the identical relation

$$
\sum_{i}^{0} x_{i}^{2} \equiv(x x)=0 .
$$

A sphere $(y)$ will be given by a relation

$$
\sum_{i}^{0 \ldots 4} y_{i} x_{i} \equiv(y x)=0
$$

and we regard the coefficients $y_{0}, \cdots y_{4}$ as the coördinates of this sphere. When $(y)$ satisfies the fundamental identity (1) the sphere is said to be null, and the point $(y)$ is called its vertex. We thus use the same notation for point and sphere coördinates, the point being considered merely as the vertex of a null sphere. The angle of two not null spheres $(x)$ and $(y)$ will be

$$
\theta=\cos ^{-1} \frac{(x y)}{\sqrt{(x x)} \sqrt{(y y)}} .
$$

Every conformal transformation of our space will be given by an orthogonal substitution in our coördinates $(x)$.

Suppose that we have four spheres $(x)(y)\left(x^{\prime}\right)\left(y^{\prime}\right)$. The coördinates of their common orthogonal spheres are given by the four-rowed determinants $\rho r_{i}=\left|x_{j} y_{k} x_{l}^{\prime} y_{m}^{\prime}\right|$. This is indeterminate when, and only when, the four spheres are linearly dependent. Assuming that no two of them are identical, this means that some sphere through the circle determined by $(x)(y)$ is also a sphere through a circle of $\left(x^{\prime}\right)\left(y^{\prime}\right)$.

Theorem 1': The necessary and sufficient condition that two circles should have more than one common orthogonal sphere is that they should be cospherical.

Theorem 2': If a circle be cospherical with each of two others which are not cospherical with one another, it is orthogonal to their common orthogonal sphere.

This second theorem comes at once from the fact that if two spheres be orthogonal to a third, so is their circle of intersection.

Through each not null circle will pass two distinct null spheres. Their vertices are called the foci of the circle and serve to determine it; the circle is the locus of points of intersection of isotropic lines through the foci. Every

* The earliest systematic treatment of pentaspherical coördinates was that of DARBOUX, Sur une classe remarquable de courbes et de surfaces, 1873. 
sphere through the foci of a not null circle cuts it orthogonally, every sphere orthogonal to such a circle passes through its foci. If two circles be so related that the first lies on a sphere through the foci of the second, then a sphere through the second and one focus of the first is orthogonal to the given sphere, and so contains the second focus of the first:

Theorem 3': If two circles be so related that one is orthogonal to a sphere through the other, then the second is orthogonal to a sphere through the first.

Two such circles are said to be in involution.

THEOREM 4': The necessary and sufficient condition that two cospherical circles should be in involution is that they should intersect orthogonally.

The proof given above for theorem $3^{\prime}$ holds when one circle lies on more than one sphere orthogonal to the other, i. e., when it contains the foci of the latter.

TheOREM 5': If two circles be so related that two spheres through one are orthogonal to the other, then every sphere through either circle is orthogonal to the other circle and the foci of each lie on the other.

Two distinct circles which bear the relation of the last theorem are said to be in bi-involution.* The figure which they form shall be called a proper cross. If a circle be null, consisting of two distinct isotropic lines, any circle orthogonal to the pencil of tangent spheres through the null circle will be in bi-involution with the null circle; together they shall be said to form an improper cross of the first sort. If a null circle consist of two identical isotropic lines, every sphere through it is null; it will be in bi-involution with itself, and be said to form an improper cross of the second sort. The following theorem is immediate.

Theorem 6: There is a single circle in bi-involution with a given circle and orthogonal to any given not null sphere which is orthogonal to the given circle.

THEOREM 7': The necessary and sufficient condition that two cospherical not null circles should intersect orthogonally is that they should be in involution.

Theorem 8: A circle cospherical with both circles of a proper cross cuts them both orthogonally.

This is an immediate result of $2^{\prime}$ and the definition of circles in bi-involution. We proceed to investigate how many circles there are, cospherical and orthogonal to each of two given not null circles. Let the first be given by its foci $(x)(y)$ while the second has foci $\left(x^{\prime}\right)\left(y^{\prime}\right)$. A circle cospherical and orthogonal to both shall be determined by the spheres $\lambda(x)+\mu(y), \lambda^{\prime}\left(x^{\prime}\right)+\mu^{\prime}\left(y^{\prime}\right)$. Since mutually perpendicular spheres are harmonically separated by the null spheres whose vertices are their foci, our two spheres above are orthogonal

\footnotetext{
*The term "involution" and "bi-involution" are taken from an admirable article by Kormas, Contributions d la theorie du cercle dans l'espace," Annales de la Faculte dos Sciences de Toulouse, vol. $2,1888$.
} 
to $\lambda(x)-\mu(y), \lambda^{\prime}\left(x^{\prime}\right)-\mu^{\prime}\left(y^{\prime}\right)$. We thus get the equations

$$
\lambda \lambda^{\prime}\left(x x^{\prime}\right)-\mu \mu^{\prime}\left(y y^{\prime}\right)=\lambda \mu^{\prime}\left(x y^{\prime}\right)-\mu \lambda^{\prime}\left(y x^{\prime}\right)=0 .
$$

Eliminating $\lambda^{\prime}: \mu^{\prime}$, we have

$$
\lambda^{2}\left(x x^{\prime}\right)\left(x y^{\prime}\right)-\mu^{2}\left(x y^{\prime}\right)\left(y x^{\prime}\right)=0 .
$$

The discriminant of this equation is

$$
4\left(x x^{\prime}\right)\left(y y^{\prime}\right)\left(x y^{\prime}\right)\left(y x^{\prime}\right) .
$$

Theorem 9: Two not null and non-cospherical circles are cospherical and orthogonal to two circles of a proper cross, and to no others when, and only when, no focus of one lies on an isotropic with a focus of the other.

Let us consider these equations in more detail. If

$$
\left(x x^{\prime}\right)=0, \quad\left(y y^{\prime}\right)\left(x y^{\prime}\right)\left(y x^{\prime}\right) \neq 0 .
$$

The isotropic $(x)\left(x^{\prime}\right)$ is the only circle fulfilling the conditions. If

$$
\left(x x^{\prime}\right)=\left(y x^{\prime}\right)=0, \quad \mu^{\prime}=0 .
$$

Here one circle passes through one focus of the other, and there are $\infty^{1}$ null circles fulfilling the conditions. If

$$
\left(x x^{\prime}\right)=\left(y y^{\prime}\right)=0,
$$

each focus of one is on an isotropic with one focus of the other, and the two circles meet two common generators of their common orthogonal sphere. These two generators must belong to the same system, as otherwise the circles would be tangent and cospherical; the circles are said in this case to be paratactic.* They are invariant under a one parameter continuous group of spherical transformations which leave invariant these two isotropic lines, as well as all those of the other system on the common orthogonal sphere. Paratactic circles will exist even in the limiting case where this sphere is null. as we see by inverting two paratactic lines of non-euclidean space. If

$$
\left(x x^{\prime}\right)=\left(y y^{\prime}\right)=\left(y x^{\prime}\right)=0, \quad\left(x y^{\prime}\right) \neq 0,
$$

we have a case essentially like the last but one. If

$$
\left(x x^{\prime}\right)=\left(x y^{\prime}\right)=\left(y x^{\prime}\right)=\left(y y^{\prime}\right)=0 .
$$

The circles are in bi-involution and have $\infty^{2}$ common cospherical and orthogonal circles.

* The concept of paratactic circles appears to be new, although coming directly from that of paratactic or Clifford parallel lines of non-euclidean space. In fact the whole question of circles cospherical with and orthogonal to two given circles seems never to have been adoquately discussed. 
ThEorem 10: The necessary and sufficient condition that tiwo circles should have but one common cospherical and orthogonal circle is that they should be noncospherical, and that a single focus of one should be on an isotropic with a single focus of the other.

THEOREM 11: The necessary and sufficient condition that two circles not null or cospherical should be cospherical and orthogonal to a singly infinite system of circles is that they should be paratactic, in which case these circles are not null, or that one focus of thc one should be on an isotropic with each.focus of the other, when the $\infty^{1}$ circles are all null.

ThEOREM 12: The necessary and sufficient condition that two not null circles should be cospherical and orthogonal to the same two parameter family of circles is that they should be in bi-involution.

Two circles are said to be in general position when the only circles cospherical and orthogonal to them are those of a proper cross. We shall define as the angles of two circles in general position those formed by the pairs of spheres through them, and through the two circles cospherical and orthogonal to them. These angles have definite values in the general case, and in the case of paratactic circles, otherwise they are null or indeterminate. Reverting to our previous notations, we have as expressions for our angles

(4) $\cos ^{2} \theta_{1}+\cos ^{2} \theta_{2}=\frac{2\left[\left(x x^{\prime}\right)\left(y y^{\prime}\right)+\left(y x^{\prime}\right)\left(x y^{\prime}\right)\right]}{(x y)\left(x^{\prime} y^{\prime}\right)}, \cos ^{2} \theta_{1} \cos ^{2} \theta_{2}=\frac{\left|\begin{array}{l}\left(x x^{\prime}\right)\left(x y^{\prime}\right) \\ \left(y x^{\prime}\right)\left(y y^{\prime}\right)\end{array}\right|^{2}}{(x y)^{2}\left(x^{\prime} y^{\prime}\right)^{2}}$.

THEOREM 13: The necessary and sufficient condition that two circles should be paratactic is that their angles should be equal, but not null or indeterminate.

Suppose that we have two paratactic circles, and a third circle cospherical with both. We assume that the common orthogonal sphere is not null. The one parameter continuous group under which the paratactic circles are invariant will carry the third circle into a series of circles cospherical with the original two. All circles of this series must intersect the same two generators of the other system of the common orthogonal sphere, since all of these generators are invariant under the group; hence all circles of the series are paratactic. They will determine a second one parameter group, and series of circles containing, among others, the original two paratactic circles. This same configuration will hold even when the sphere is null, as we see by continuity, or by inverting the circles into lines. Remembering, lastly, that the only surfaces which have a double generation by means of circles, where each circle of one generation is cospherical with each of the other, are cyclides, we have

ThEOREM 14: Two paratactic circles belong to a singly infinite system of cyclides each having two conjugate generations made up of paratactic circles. 


\section{§ 2. Circles Orthogonal to One Sphere.*}

Let us next turn our attention particularly to those circles which are all orthogonal to one not null sphere; those orthogonal to a null sphere are obtained by inversion from straight lines. We may freely assume that the fundamental sphere is $x_{5}=0$. We determine each circle of the system then by its six Plücker coördinates

$$
\rho p_{0 i}=x_{0} y_{i}-x_{i} y_{0}, \quad \rho p_{j k}=x_{j} y_{k}-x_{k} y_{j} \quad(j \neq 0, k \neq 0) .
$$

It is clear that these coördinates, being supposed homogeneous, are unaltered if $(x)$ and $(y)$ be replaced by any two of their linear combinations so that our ccördinates depend upon the circle and not on the two particular spheres through it. Two circles of our system will be cospherical when they have a common orthogonal sphere other than the fundamental one; the necessary and sufficient condition for this is

$$
p_{01} q_{23}+p_{02} q_{31}+p_{03} q_{12}+p_{23} q_{01}+p_{31} q_{02}+p_{12} q_{03} \equiv(p / q)=0 \text {. }
$$

We have also the fundamental identity

$$
(p / p)=0 \text {. }
$$

Conversely we prove exactly as in the Plücker line geometry that every set of homogeneous ccördinates which satisfy this last identity will correspond to a single circle orthogonal to our fundamental sphere.

There is a perfect one-to-one correspondence between the circles orthogonal to a not null sphere and the lines of projective space; cospherical circles corresponding to intersecting lines. A sphere orthogonal to the fundamental sphere will correspond to a point, a pair of points mutually inverse in the fundamental sphere will correspond to a plane. A pencil of cospherical circles will correspond to a pencil of lines, a cyclide anallagmatic in the fundamental sphere will correspond to a quadric.

When the fundamental sphere is not a plane, and the figures are looked upon as in euclidean space, this correspondence may be easily effected by referring each circle to its axis, i. e., the line through its center, orthogonal to its plane. Two circles $(p)$ and $(q)$ will be in involution if

$$
\Sigma p_{i j} q_{i j}=0 \text {. }
$$

If two circles of our system be in involution, each is cospherical with that circle which is orthogonal to the fundamental sphere, and in bi-involution with the other, and conversely. The circle of our system in bi-involution

* For an analytic treatment of this topic see the dissertation of Forbes: The Geometry of Circles Orthogonal to a Given Sphere, New York, 1904. Synthetically by C. L. E. Moore: Circles Orthogonal to One Sphere, Annals of Mathematics, Series 2, vol. 8, 1907. 
with $(p)$ will thus have the coördinates

$$
p_{0 i}^{\prime}=\rho p_{j k}, \quad p_{j k}^{\prime}=\rho p_{0 i} .
$$

The necessary and sufficient condition that two circles of our system should be cospherical and orthogonal is

$$
(p / q)=\Sigma p_{i j} q_{i j}=0 .
$$

The only circles in involution with themselves are null circles; the condition here is

$$
\Sigma p_{i j}^{2}=0 .
$$

The fundamental invariant of two not null circles is the product of the cosines of their angles; $*$ this, by (4), has the value

$$
I(p q) \equiv \cos \theta_{1} \cos \theta_{2}=\frac{\Sigma p_{i j} q_{i j}}{\sqrt{\Sigma p_{i j}^{2}} \sqrt{\Sigma q_{i j}^{2}}} .
$$

The necessary condition that two circles should be paratactic is

$$
\left\{\left[(p / q)+\Sigma p_{i j} q_{i j}\right]^{2}-\Sigma p_{i j}^{2} \Sigma q_{i j}^{2}\right\}\left\{\left[(p / q)-\Sigma p_{i j} q_{i j}\right]^{2}-\Sigma p_{i j}^{2} \Sigma q_{i j}^{2}\right\}=0 \text {. }
$$

A system of circles orthogonal to our fundamental sphere and satisfying an equation of the type

$$
\Sigma a_{i j} p_{i j}=0
$$

is called a "linear complex." If

$$
(a / a)=0
$$

the complex is special, and is composed of the assemblage of all circles cospherical with a given circle, if not we have a rather more complicated system. The circles orthogonal to our fundamental sphere, and in bi-involution with those of our complex (11) belong to a second linear complex, whose equation is

$$
(a / p)=0 .
$$

The congruence of circles common to these two (in the general case where equations (11) and (12) are not equivalent to one another) are all cospherical with the two circles

$$
\begin{gathered}
\lambda a_{0 i}+\mu a_{j k}, \\
\left(\lambda^{2}+\mu^{2}\right)(a / a)+2 \lambda \mu \Sigma a_{i j}^{2}=0 .
\end{gathered}
$$

Our linear complex shall be said to be general when these two are distinct. We call them $(\alpha)$ and $\left(\alpha^{\prime}\right)$ and note that they are in bi-involution, since

* This invariant is due to Kosnigs, loc. cit., p. 79, i. e., he gives the same analytic form, but a different interpretation. A neat interpretation as the cosine of a single angle has recently been found by Mr. David Barrow of Harvard University. 
equations (11) and (12) are merely interchanged when each Plücker coördinate is replaced by its complementary. We also see that we may write

$$
\begin{gathered}
\alpha_{i j}=\lambda a_{i j}+\mu a_{k l}, \quad \alpha_{i j}^{\prime}=\lambda a_{k l}+\mu a_{i j}, \\
\frac{I\left(\alpha^{\prime} p\right)}{I(\alpha p)}=\frac{\lambda(a / p)+\mu \Sigma a_{i j} p_{i j}}{\lambda \Sigma a_{i j} p_{i j}+\mu(a / p)} .
\end{gathered}
$$

If, therefore, $(p)$ be a line of our linear complex, we have

$$
\frac{I\left(\alpha^{\prime} p\right)}{I(\alpha p)}=\frac{\lambda}{\mu}=-\frac{\Sigma a_{i j}^{2}+\sqrt{\left(\Sigma \dot{a}_{i j}^{2}\right)^{2}-(a / a)^{2}}}{(a / a)}
$$

THEOREM 15: The general linear complex of circles orthogonal to a not null sphere is composed of all circles whose fundamental invariants with the two circles of a proper cross orthogonal to the sphere bear to one another a fixed ratio whose square is not unity and, conversely, every such assemblage is a linear complex.

The circles $(\alpha)$ and $\left(\alpha^{\prime}\right)$ are called the axial circles of the complex, our theorem will hold even when the fundamental sphere is null, as we see by continuity, or by inversion from the non-euclidean line geometry.*

We shall confine our discussion in the present section to the general linear complex and not enter into any special case except the following. If

and if we put

$$
a_{0 i}=\rho a_{j k}, \quad a_{j k}=\rho a_{0 i}, \quad \rho^{2}=1,
$$

$$
q_{i j}^{\prime}=(a / a) q_{i j}-2\left(\Sigma a_{i j} q_{i j}\right) a_{i j},
$$

where $(q)$ is any circle orthogonal to the fundamental sphere, but not belonging to our complex, then

$$
\begin{gathered}
(a / a)=\Sigma a_{i j}^{2}, \quad \Sigma q_{i j}^{\prime 2}=(a / a)^{2} \Sigma q_{i j}^{2}, \\
\frac{I\left(q^{\prime} / p\right)}{I(q / p)}=1 .
\end{gathered}
$$

If we choose our coördinate system so that the axial circles are $x_{0}=x_{1}=0$; $x_{2}=x_{3}=0$ our complex becomes

$$
a_{01} p_{01}+a_{23} p_{23} \text {. }
$$

If a general linear complex be given, the axial circles are given, the converse is not true. We may, in fact, replace our complex $(a)$ by $\left(a^{\prime}\right)$, where

$$
a_{i j}^{\prime}=\lambda a_{i j}+\mu a_{k l} \text {. }
$$

The equations to determine the axial circles are replaced by simple linear combinations of themselves, and the axial cross is as before. We are thus led

\footnotetext{
- See the author: The Metrical Aspect of the Line-Sphere Transformation, these T r a n s a c t i o n B, vol. 12, 1911.
} 
to a simple set of coördinates for this cross *

$$
a_{0 i}+a_{j k}=\rho X_{i}, \quad a_{0 i}-a_{j k}=\sigma \dot{X}_{i} .
$$

If a general linear complex be given, the triads of separately homogeneous coordinates are immediately found, and it is easily seen that they are merely affected by constant multipliers if the complex be replaced by another with the same axial circles. Conversely, when we have the two sets of separately homogeneous coördinates $(X)(\dot{X})$ with the limitation

the linear complex

$$
\left(\Sigma X_{i}^{2}\right)\left(\Sigma \dot{X}_{i}^{2}\right) \neq 0,
$$

$$
2 a_{0 i}=X_{i}+\dot{X}_{i}, \quad 2 a_{j k}=X_{i}-\dot{X}_{i}
$$

will be a general complex, whose axial cross has the coördinate values $(X)(\dot{X})$.

Theorem 16: The assemblage of all proper crosses orthogonal to a not null sphere may be put into one to one correspondence with the assemblage of all pairs of points of two projective planes not lying on two specified conics, one in each plane.

We may, with a little ingenuity, extend our correspondence so as to include points of these exiled conics, we shall not however pause to do so here. If two circles have the coördinates $(a)$ and $(b)$, we shall find

$$
\begin{aligned}
\cos \theta_{1} \cos \theta_{2}=\frac{\Sigma a_{i j} b_{i j}}{\sqrt{\Sigma a_{i j}^{2}} \sqrt{\bar{\Sigma} b_{i j}^{2}}}, & \sin \theta_{1} \sin \theta_{2}=\frac{(a / b)}{\sqrt{\Sigma a_{i j}^{2} \sqrt{\Sigma b_{i j}^{2}}}}, \\
\cos \left(\theta_{1} \pm \theta_{2}\right)=\frac{\Sigma X_{i} X_{i}^{\prime}}{\sqrt{\overline{\Sigma X_{i}^{2}} \sqrt{\Sigma X_{i}^{\prime \prime}}},} & \cos \left(\theta_{1} \mp \theta_{2}\right)=\frac{\Sigma \dot{X}_{i} \dot{X}_{i}^{\prime}}{\sqrt{\Sigma X_{i}^{2}} \cdot \overline{\Sigma X_{i}^{\prime 2}}} .
\end{aligned}
$$

The condition that two proper circles shall be cospherical, or that each should be cospherical with the circle of our system in bi-involution with the other is

$$
\frac{\Sigma X_{i} X_{i}^{\prime}}{\sqrt{\Sigma \overline{X_{i}^{2}} \sqrt{\Sigma X_{i}^{\prime 2}}}}= \pm \frac{\Sigma \dot{X}_{i} \dot{X}_{i}}{\sqrt{\Sigma \dot{X}_{i}^{2}} \sqrt{\overline{\Sigma \dot{X}_{i}^{\prime 2}}}} \text {. }
$$

Two circles orthogonal to our fundamental sphere are cospherical and orthogonal if

$$
\Sigma X_{i} X_{i}^{\prime}=\Sigma \dot{X}_{i} \dot{X}_{i}^{\prime}=0 .
$$

It will be convenient to say in the present case that the two crosses intersect orthogonally. The necessary conditions for parataxy become

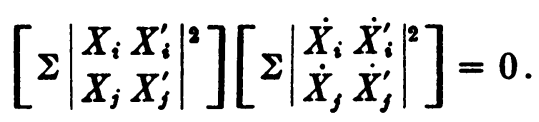

- See the author's Non-Euclidean Geometry, cit. Ch. XII. The developments of the present section follow so closely those previously established that we give them here in all brevity. 
The necessary and sufficient conditions are thus

$$
\rho X_{i}^{\prime}=X_{i} \text { or } \sigma \dot{X}_{i}^{\prime}=\dot{X}_{i} .
$$

The simplest one parameter families of circle crosses are those whose coördinates are linearly dependent upon those of two fixed crosses,

$$
X_{i}=\lambda Y_{i}+\mu Z_{j}, \quad \dot{X}_{i}=\lambda \dot{Y}_{i}+\mu \dot{Z}_{i} .
$$

These are the axial complexes of the pencil of linear complexes

$$
a_{i j}=\lambda b_{i j}+\mu c_{i j} \text {. }
$$

These families fall under one of two decidedly different types.

$$
\rho Y_{i} \equiv Z_{i}, \quad \sigma \dot{Y}_{i} \neq \dot{Z}_{i} .
$$

Here all circles of the series are paratactic. Let us determine $\left(\dot{X}^{\prime}\right)$ from the equations

then

$$
\Sigma \dot{X}_{i}^{\prime} \dot{Y}_{i}=\Sigma \dot{X}_{i}^{\prime} \dot{Z}_{i}=0,
$$

$$
\Sigma \dot{X}_{i}^{\prime} \dot{X}_{i}=0 \text {. }
$$

On the other hand if $\left(X^{\prime}\right)$ merely satisfy the equations

$$
\Sigma Y_{i} X_{i}^{\prime}=0
$$

the crosses $\left(\dot{X}^{\prime}\right)\left(\dot{X}^{\prime}\right)$ generate a similar system, and every cross $(X)(\dot{X})$ cuts every cross $\left(X^{\prime}\right)\left(\dot{X}^{\prime}\right)$ orthogonally. The circles of our series generate a cyclide.

$$
\begin{array}{cc}
\rho Y_{i} \neq Z_{i}, & \sigma \dot{Y}_{i} \neq \dot{Z}_{i}, \\
U_{i}=Y_{j} Z_{k}-Y_{k} Z_{j}, & \dot{U}_{i}=\dot{Y}_{i} \dot{Z}_{k}-\dot{Y}_{k} \dot{Z}_{j}, \\
\Sigma U_{i} X_{i}=\Sigma \dot{U}_{i} \dot{X}_{i}=0 .
\end{array}
$$

All crosses of our series cut one fixed cross orthogonally. The surface so generated, even in degenerate cases, shall be a called a pseudo-cylindroid.* It has one general and very fundamental property. Suppose that we have an analytic congruence of circles orthogonal to our fundamental sphere

$$
X_{i}=X_{i}(u v), \quad \dot{X}_{i}=\dot{X}_{i}(u v) .
$$

Let us further assume

$$
\left|X \frac{\partial X}{\partial u} \frac{\partial X}{\partial v}\right| \neq 0, \quad\left|\dot{X} \frac{\partial \dot{X}}{\partial u} \frac{\partial \dot{X}}{\partial v}\right| \neq 0 .
$$

This means that our circles can not all be assembled into one parameter families of paratactic circles. We mean by a circle in general position in our congruence one whose coördinates will not make the left sides of the above

- From the analogous figure in line-geometry, the euclidean or non-euclidean cylindroid. 
expressions vanish. We have

Theorem 17: If an analytic congruence of circles be given orthogonal to one sphere, whose members can not be all assembled into one parameter paratactic families, and if a circle be taken in general position in such a congruence, then the circles cospherical and orthogonal to this and to the infinitely near circles of the congruence will generate a pseudo-cylindroid.

It is time to hunt for a canonical form for our pseudo-cylindroid. We may take for the cross which all cut orthogonally

$$
\left(\begin{array}{lll}
1 & 0 & 0
\end{array}\right)\left(\begin{array}{lll}
1 & 0 & 0
\end{array}\right) \text {. }
$$

Two crosses of the series will intersect orthogonally, we have but to solve the equations

$$
\begin{aligned}
& \lambda \lambda^{\prime} \Sigma Y_{i} Y_{i}+\left(\lambda \mu^{\prime}+\mu \lambda^{\prime}\right) \Sigma Y_{i} Z_{i}+\mu \mu^{\prime} \Sigma Z_{i} Z_{i} \\
& =\lambda \lambda^{\prime} \Sigma \dot{Y}_{i} \dot{Y}_{i}+\left(\lambda \mu^{\prime}+\mu \lambda^{\prime}\right) \Sigma \dot{Y}_{i} \dot{Z}_{i}+\mu \mu^{\prime} \Sigma \dot{Z}_{i} \dot{Z}_{i}=0 .
\end{aligned}
$$

Eliminating $\lambda^{\prime}: \mu^{\prime}$, we get a quadratic in $\lambda: \mu$; its discriminant is the simultaneous invariant of

$$
\Sigma X_{1}^{2}=0, \quad \Sigma \dot{X}_{i}^{2}=0,
$$

when these are written as quadratics in $\lambda: \mu$. When our crosses have real coördinates the roots of each of these two quadratic equations are conjugate imaginary pairs of values, and two such pairs can not separate one another harmonically; hence the simultaneous invariant can not vanish, and there are usually two distinct crosses of the series cutting one another orthogonally. Taking these for $\left(\begin{array}{lll}0 & 1 & 0\end{array}\right)\left(\begin{array}{lll}0 & 1 & 0\end{array}\right)$ and $\left(\begin{array}{lll}0 & 0 & 1\end{array}\right)\left(\begin{array}{lll}0 & 0 & 1\end{array}\right)$ we may reduce the equations of our series to

$$
\rho X_{1}=\alpha \dot{X}_{1}=0, \quad \rho X_{2}=\beta \dot{X}_{2}, \quad \rho X_{3}=\gamma \dot{X}_{3} \quad(\alpha, \beta, \gamma, \text { const. }) .
$$

To find the point equation of the surface let $(p)$ be the Plücker coördinates of a circle of one of our crosses;

$$
\begin{aligned}
p_{01} & =p_{23}=0, \\
\beta\left(p_{02}-p_{31}\right)\left(p_{03}+p_{12}\right) & =\gamma\left(p_{03}-p_{12}\right)\left(p_{02}+p_{31}\right) .
\end{aligned}
$$

A point $(x)$ will be on this circle if

$$
x_{2} p_{02}+x_{3} p_{03}=x_{0} p_{02}+x_{1} p_{i 2}=0 .
$$

Eliminating from these equations and the identity (6) we have

$$
\beta\left(x_{0} x_{2}+x_{3} x_{1}\right)\left(x_{0} x_{3}+x_{1} x_{2}\right)+\gamma\left(x_{3} x_{1}-x_{0} x_{2}\right)\left(x_{1} x_{2}-x_{0} x_{3}\right)=0 .
$$

Theorem 18: The non-degenerate pseudo-cylindroid is a surface of the eighth order. 
Among congruences of crosses the most interesting is the chain congruence which consists of crosses whose coördinates are linearly dependent on three of their number, or the axial crosses of a net of linear complexes. We write in this case

$$
X_{i}=a Y_{i}+b Z_{i}+c T_{i}, \quad \dot{X_{i}}=a \dot{Y_{i}}+b \dot{Z_{i}}+c \dot{T_{i}} .
$$

Various sub-cases must be noticed.

$$
|Y Z T|=|\dot{Y} \dot{Z} \dot{T}|=0 .
$$

Assuming that we have surely a two parameter family of crosses, the first minors can not all vanish in either of these determinants. We have the congruence of crosses cutting a given cross orthogonally,

$$
\begin{aligned}
& \text { 1. } Y Z T|=0, \quad| \dot{Y} \dot{Z} \dot{T} \mid \neq 0, \\
& T_{i}=\lambda Y_{i}+\mu Z_{i}, \quad X_{i}=(a+c \lambda) Y_{i}+(b+c \mu) Z_{i} .
\end{aligned}
$$

$$
\begin{gathered}
\frac{a+c \lambda}{b+c \mu}=\frac{p}{q}=\text { const. } \\
c=\frac{b p-a q}{q \lambda-p \mu}, \\
X_{i}=p Y_{i}+q Z_{i}, \quad \dot{X}_{i}=a\left[\dot{Y}_{i}-\frac{q \dot{T}_{i}}{q \lambda-p \mu}\right]+b\left[Z_{i}+\frac{p \dot{T}_{i}}{q \lambda-p \mu}\right] .
\end{gathered}
$$

The crosses may be grouped into paratactic generations of $\infty^{1}$ cyclides.

$$
|Y Z T| \cdot|\dot{Y} \dot{Z} \dot{T}| \neq 0 \text {. }
$$

Solving the equations (15) for $a, b, c$, we have

$$
\rho \dot{X}_{i}=\Sigma_{j} a_{i j} X_{j} .
$$

The crosses intersecting orthogonally pairs of crosses of our congruence will generate the conjugate congruence which is of like structure,

$$
\sigma U_{i}=\Sigma_{j} A_{i j} U_{j}
$$

The relation between these two congruences is reciprocal, each is made up of the crosses intersecting orthogonally pairs of crosses of the other. Let us see if we can find any crosses which belong simultaneously to the two conjugate congruences. For such an one

$$
\tau X_{i}=\Sigma_{j} b_{i j} X_{j}
$$

These equations will yield a cubic equation in $\tau$. Let us say that the chain congruence is general when this equation has three distinct roots. Each root gives a cross common to the two congruences, the common perpendicular 
to each two of these crosses must be the third. Each two must intersect orthogonally. We may then take them as the basis of our coördinate system,. namely the crosses

$$
\left(\begin{array}{lll}
1 & 0 & 0
\end{array}\right)\left(\begin{array}{lll}
1 & 0 & 0
\end{array}\right), \quad\left(\begin{array}{lll}
0 & 1 & 0
\end{array}\right)\left(\begin{array}{lll}
0 & 1 & 0
\end{array}\right), \quad\left(\begin{array}{lll}
0 & 0 & 1
\end{array}\right)\left(\begin{array}{lll}
0 & 0 & 1
\end{array}\right) .
$$

The general chain congruence and its conjugate will then appear in the canonical form

$$
\begin{gathered}
\rho \dot{X}_{i}=a_{i} X_{i}, \quad \sigma U_{i}=a_{i} \dot{U}_{i}, \\
a_{1} a_{2} a_{3}\left(a_{1}-a_{2}\right)\left(a_{2}-a_{3}\right)\left(a_{3}-a_{1}\right) \neq 0 .
\end{gathered}
$$

The condition that two crosses of our conjugate congruences should be cospherical or that one should be cospherical with the circle in bi-involution with the other is

$$
\Sigma U_{i} X_{i}\left[\sqrt{\Sigma a_{i} X_{i}^{2}} \sqrt{\Sigma \frac{1}{a_{i}} \cdot U_{i}^{2}} \pm v^{\prime} \overline{\Sigma X_{i}^{2}} \sqrt{\Sigma U_{i}^{2}}\right]=0 .
$$

If the first factor vanish, then the cospherical circles cut orthogonally, as do their mates. If the second factor vanish, one circle of each cross of the first congruence for which

$$
q \Sigma a_{i} X_{i}^{2}=p \Sigma X_{i}^{2}
$$

is cospherical with one circle of each cross of the second congruence for which

$$
p \Sigma \frac{1}{a_{i}} \cdot U_{i}^{2}=q \Sigma U_{i}^{2} .
$$

TheOREM 19: The circles of a general chain congruence cospherical with a circle of the conjugate congruence and orthogonal to it generate a pseudo-cylindroid, those which are cospherical but not orthogonal to such a circle generate a cyclide; the conjugate generation of this cyclide belongs to the conjugate congruence.

If a cyclide have our fundamental sphere as one of its spheres of inversion, it is clear that the circles of either corresponding generation can be rationally expressed in the form

$$
X_{i}=t^{2} Y_{i}+2 t Z_{i}+T_{i}, \quad \dot{X}_{i}=t^{2} \dot{Y}_{i}+2 t \dot{Z}_{i}+\dot{T}_{i} .
$$

Theorem 20: The circles cospherical and orthogonal to pairs of circles of one generation of a cyclide generate a chain congruence which includes the conjugate generation of the same cyclide.

Taking the equation of our general chain congruence in the form (16) we may assume that the sphere $x_{0}=0$ bears no special relation to the congruence. All circles of this sphere satisfy the relation

$$
\dot{X}_{i}=\rho X_{i} .
$$

Substituting in (16) we have a cubic in $\rho$. 
ThEOREM 21: Three circles of a general chain congruence lie on an arbitrary sphere orthogonal to the fundamental sphere, and three also of the conjugate congruence. The triangles formed by the first three circles are polars to those formed by the second three.

Our congruence is made up of pairs of circles in bi-involution. If a circle lie on a sphere orthogonal to the fundamental sphere, its mate passes through the foci of the circle common to these two spheres.

THEOREM 22: Through each pair of points which are mutually inverse with regard to the fundamental sphere will pass three circles of a general chain congruence, and three of the conjugate congruence. Each circle of one triad cuts two of the other orthogonally.

\section{§3. Circles in Space.}

We may determine a circle in general position in pentaspherical space by Plücker coördinates exactly as we did in the special case when it was orthogonal to a given sphere, albeit the formulæ are somewhat more complicated in the general case. If our circle be given by the spheres $(x)$ and $(y)$, we shall write

$$
\rho p_{i j}=x_{i} y_{j}-x_{j} y_{i} .
$$

Of these coördinates there are ten which are essentially distinct, and when we use the summation sign we shall mean to cover ten only. For some purposes it is better to let both $i$ and $j$ take any one of the five values, writing the additional equations

$$
p_{i j}=-p_{j i}, \quad p_{i i}=0 .
$$

It is evident that as the assemblage of all circles depends upon but six parameters the Plücker coördinates must be connected by certain identical relations, and this is the case, for there are five fundamental identities, which we write at length, largely to establish a convention of sign.

$$
\begin{aligned}
& \frac{1}{2} \Omega_{0}(p p)=p_{12} p_{34}+p_{13} p_{42}+p_{14} p_{23}=0, \\
& \frac{1}{2} \Omega_{1}(p p)=p_{02} p_{43}+p_{04} p_{32}+p_{03} p_{24}=0, \\
& \frac{1}{2} \Omega_{2}(p p)=p_{01} p_{34}+p_{03} p_{41}+p_{04} p_{13}=0, \\
& \frac{1}{2} \Omega_{3}(p p)=p_{01} p_{42}+p_{04} p_{21}+p_{02} p_{14}=0, \\
& \frac{1}{2} \Omega_{4}(p p)=p_{01} p_{23}+p_{02} p_{31}+p_{03} p_{12}=0 .
\end{aligned}
$$

The polarized form for $\Omega_{i}(p p)$ shall be written $\Omega_{i}(p q) .^{*}$ It is clear that these identities can not all be independent as then we should have but $\infty 4$ circles. In fact if $(p)$ be any system of homogeneous values satisfying

- The credit for introducing these coördinates for a circle seems to be due to Strpannos. See his note Sur une configuration remarquable de quinze cercles dans lespace, Co m p t e s Rend u 8 , Vol. 83 (1882). 
merely (20) we shall have

$$
\sum_{n} p_{\text {in }} \Omega_{n}(p p) \equiv 0
$$

If $p_{i j} \neq 0$, every set of values which satisfy

will also satisfy

$$
\Omega_{k}(p p)=\Omega_{l}(p p)=\Omega_{m}(p p)=0
$$

$$
\Omega_{i}(p p)=\Omega_{j}(p p)=0 .
$$

Every circle will thus have ten homogeneous and distinct coördinates satisfying our equations (21). Conversely if any set of homogeneous values, not all zero be given which satisfy $(20)$ and (21), they will be the Plücker coördinates of just one circle. The proof is straightforward, and perfectly well known.*

It is occasionally advantageous to determine a circle, not by two spheres through it, but by three spheres to which it is orthogonal. Let these be the spheres $(r)(s)(t)$. If $(u)$ and $(v)$ be arbitrary spheres, we may imagine that our circle lies on the spheres $(x)$ and $(y)$, where

$$
x_{i}=\left|r_{j} s_{k} t_{l} u_{m}\right|, \quad y_{i}=\left|r_{j} s_{k} t_{l} v_{m}\right| ;
$$

substituting in (19), we get by a well-known theorem in determinants

$$
p_{i j}=\Delta\left|\begin{array}{lll}
r_{k} & r_{l} & r_{m} \\
s_{k} & s_{l} & s_{m} \\
t_{k} & t_{l} & t_{m}
\end{array}\right| \text {. }
$$

The condition that the two circles $(p)$ and $(q)$ be in involution is easily found to be

$$
\Sigma p_{i j} q_{i j}=0 \text {. }
$$

We get from the invariant equation (9),

Two circles will be cospherical if

$$
I(p, q)=\frac{\Sigma p_{i j} q_{i j}}{\sqrt{\Sigma p_{i j}^{2}} \sqrt{\Sigma q_{i j}^{2}}}
$$

$$
\Omega_{i}(p q) \equiv 0 .
$$

The common orthogonal sphere to two non-cospherical circles $(p)$ and $(q)$ is

$$
\rho r_{i}=\Omega_{i}(p q) \text {. }
$$

Two spheres will have a common point if this sphere be null, i. e., if

$$
\Sigma \Omega_{i}^{2}(p q)=0 \text {. }
$$

If $(x)$ and $(y)$ be the foci of $(p)$, and $(q)$ be in bi-involution therewith, we may * See, e. g., Komnas, loc. cit. 
write

$$
q_{i j}=\left|\begin{array}{lll}
x_{k} & x_{l} & x_{m} \\
y_{k} & y_{l} & y_{m} \\
t_{k} & t_{l} & t_{m}
\end{array}\right| .
$$

The condition that two circles be in bi-involution is thus

$$
\Sigma_{n} p_{\text {in }} q_{\text {in }} \equiv 0 \text {. }
$$

The necessary condition that two circles be paratactic is

$$
\left[\Sigma p_{i j}^{2} \Sigma q_{i j}^{2}+\left(\Sigma p_{i j} q_{i i}\right)^{2}-\Sigma \Omega_{i}^{2}(p q)\right]^{2}=4\left(\Sigma p_{i j} \Sigma_{i j}\right)^{2} \Sigma p_{i j}^{2} \Sigma q_{i j}^{2} \text {. }
$$

We see, in fact, that this is an invariant condition for all quinary orthogonal substitutions, and it reduces to (11) when the common orthogonal sphere is $x_{4}=0$.

A five parameter family of circles shall be called a hypercomplex. The simplest type of hypercomplex is the linear one, given by an equation of the form

$$
\Sigma a_{i j} p_{i j}=0 .
$$

As the sphere $x_{4}=0$ may be supposed to bear no special relation to this hypercomplex, we have

THEOREM 23'. The circles of a linear hypercomplex orthogonal to an arbitrary sphere generate a linear complex.

Theorem 24'. The assemblage of all circles in involution with a given circle is a linear hypercomplex.

The condition that our hypercomplex (30) should be of this latter type is manifestly

$$
\boldsymbol{\Omega}_{i}(a a) \equiv \mathbf{0} .
$$

Let us now suppose that this condition is not fulfilled. Our hypercomplex will have two fundamental invariants of Koenigs which we write

$$
\Sigma a_{i j}^{2}=I, \quad \Sigma \Omega_{i}^{2}(a a)=J .
$$

If (30) be rewritten in the form

$$
\Sigma a_{i j}\left(x_{i} y_{j}-x_{j} y_{i}\right)=0
$$

then if $(y)$ be considered known, the sphere $(x)$ will be orthogonal to the sphere

$$
z_{i}=\sum_{n} a_{i n} y_{n} .
$$

It will be found immediately that

$$
\Sigma z_{n} \Omega_{n}(a a)=(y z)=0 \text {. }
$$

The circles of the hypercomplex on an arbitrary sphere are orthogonal to its 
intersection with a sphere which is orthogonal to it and to a fixed sphere

$$
r_{i}=\Omega_{i}(a a) .
$$

This latter is called the central sphere of the hypercomplex. We notice that $(z)$ disappears when $(y)$ takes the value $\Omega(a a)$ so that all circles of the central sphere belong to the hypercomplex. If $J \neq 0$ we may take as the central sphere of the hypercomplex $x_{4}=0$. We have then

$$
a_{i 4} \equiv 0 \text {. }
$$

The equation of the hypercomplex becomes then identical with (11); we may find $(\alpha)$ and $\left(\alpha^{\prime}\right)$ as in the last section, and we have the fundamental equations

$$
\frac{I\left(\alpha^{\prime} p\right)}{I(a p)}=-\frac{\Sigma a_{i j}+\sqrt{\left(\Sigma a_{i j}\right)^{2}-(a / a)^{2}}}{(a / a)}=\frac{-I+\sqrt{I^{2}-J}}{\sqrt{ } J} .
$$

We define as general any linear hypercomplex for which

$$
J\left(I^{2}-J\right) \neq 0 .
$$

THEOREM 25: The general linear hypercomplex consists in the assemblage of all circles whose fundamental invariants with the two circles of a proper cross bear to one another a fixed ratio whose square is not unity. The common orthogonal sphere to the two circles of the cross is the central sphere of the hypercomplex.*

We find for our general linear hypercomplex the canonical form

$$
\left[I+\sqrt{I^{2}-J}\right] p_{0 i}+\sqrt{J} \bar{J}_{23}=0 .
$$

Let us establish this fundamental theorem in another way, without altering the coördinate system to fit the given hypercomplex. Assuming always that our hypercomplex is general we introduce some new symbols as follows:

$$
A_{i j}=\Omega_{k}(a a) a_{l m}+\Omega_{l}(a a) a_{m k}+\Omega_{m}(a a) a_{k l} .
$$

We easily reach the following identities with the aid of (22):

If, then, we write

$$
\Omega_{i}(a A)=I \Omega_{i}(a a), \quad \Omega_{i}(A A)=J \Omega_{i}(a a),
$$

$$
\Sigma a_{i j} A_{i j}=J, \quad \Sigma A_{i j}^{2}=I J .
$$

$$
\begin{gathered}
\alpha_{i j}=x a_{i j}+A_{i j}, \\
\Omega_{i}(\alpha \alpha)=\left(x^{2}+2 x I+J\right) \Omega_{i}(a a) .
\end{gathered}
$$

Let $x_{1}$ and $x_{2}$ be the roots of the equation

$$
x^{2}+2 x I+J=0 .
$$

*This fundamental property appears to have escaped the notice of previous writers dealing with the linear hypercomplex.

Trans Am. Math. Boc. 11 
We have two circles

$$
\begin{aligned}
\alpha_{i j} & =x_{1} a_{i j}+A_{i j}, & \alpha_{i j}^{\prime} & =x_{2} a_{i j}+A_{i j} \\
\Sigma \alpha_{i j}^{2} & =2 x_{1}\left(J-I^{2}\right) & \Sigma \alpha_{i j}^{\prime 2} & =2 x_{2}\left(J-I^{2}\right) .
\end{aligned}
$$

For a circle of our given hypercomplex we shall have

Notice also that

$$
\frac{I\left(\alpha^{\prime} p\right)}{I(\alpha p)}=\frac{\sqrt{x_{1}}}{\sqrt{x_{2}}}-\frac{-I+\sqrt{I^{2}-J}}{\sqrt{J}} .
$$

$$
\begin{aligned}
\sum_{n} \alpha_{i n} \Omega_{n}(a a) & =\sum_{n} A_{i n} \Omega_{n}(a a) \\
& =\sum_{j k}\left(a_{l m}+a_{m l}\right) \Omega_{j} \Omega_{k} \\
& =0 ;
\end{aligned}
$$

the two circles $(\alpha)\left(\alpha^{\prime}\right)$ are orthogonal to the central sphere. We have also

$$
\sum_{n} \alpha_{i n} \alpha_{i n}^{\prime}=0
$$

This shows that they are in bi-involution. Our theorem is established again. We shall call these the axial circles, or circles of the axial cross of the hypercomplex. We may find $\infty^{1}$ other linear hypercomplexes with the same axial cross; we have but to write

$$
\begin{aligned}
a_{i j}^{\prime} & =p a_{i j}+\frac{q A_{i j}}{\sqrt{J}}, \\
\Omega_{i}\left(a^{\prime} a^{\prime}\right) & =\left(p^{2}+2 p q \frac{I}{\sqrt{J}}+q^{2}\right) \Omega_{i}(a a), \\
\sqrt{J^{\prime}} & =\left(p^{2}+2 p g \frac{I}{\sqrt{J}}+q^{2}\right) \sqrt{J}, \\
A_{i j}^{\prime} & =\left(p^{2}+2 p q \frac{I}{\sqrt{J}}+q^{2}\right)\left[p A_{i j}+q \sqrt{J} a_{i j}\right] .
\end{aligned}
$$

The two circles whose coördinates are linear combinations of $a_{i j}$ and $A_{i j}$ may be expressed equally well by means of a linear combination of $a_{i j}^{\prime}$ and $A_{i j}^{\prime}$.

$$
\begin{aligned}
\left(a_{i j}+\frac{A_{i j} \delta}{\sqrt{J^{\prime}}}\right) & =(p+q \delta)\left(a_{i j}+\frac{A_{i j} \delta}{\sqrt{J}}\right), \\
\delta^{2} & =1 .
\end{aligned}
$$

If, then, we introduce the complex variable

$$
(x+y \delta), \quad \delta^{2}=1,
$$

we may thus take the expressions

$$
\left(a_{i j}+\frac{A_{i j} \delta}{\sqrt{J}}\right)
$$


as homogeneous complex coördinates of our cross, for if we multiply them by any complex multiplier of the present type, the linear hypercomplex is replaced by another with the same axial cross. Conversely if a proper cross be given, there are $\infty^{1}$ linear hypercomplexes having it as axial cross (we have but to consider the case where the orthogonal sphere is $x_{4}=0$ ) and we may find for it a set of homogeneous complex coördinates of the present type. The only drawback to these coördinates is that $(a)$ and $(A)$ are not independent variables.

We must now see what becomes of the axial cross of our linear hypercomplex when the latter takes certain special forms. Let us first multiply our complex coördinates by $\sqrt{J}$, we get $\sqrt{J} a_{i j}+A_{i j} \delta$. Assuming then that

$$
\sqrt{J}=0, \quad J^{2}-I \neq 0 .
$$

The central sphere is null, equation (35) has one root $x=0$ giving the circle $(A)$ which by (34) is null; the other circle of the cross is

$$
a_{i j}^{\prime}=-2 I a_{i j}+A_{i j},
$$

and not null; we have an improper cross of the first sort. Suppose next

$$
I^{2}-J=0 .
$$

The two members of our cross fall together, so that we have an improper cross of the second sort imbedded in the central sphere, which is null if $J=0$.

Suppose thirdly that

$$
A_{i j}=\rho a_{i j}, \quad \Omega_{i}(a a) \neq 0 .
$$

The equation to determine the axial cross becomes entirely illusory, we have exactly what we had before in the indeterminate case of the linear complex.

When

$$
\Omega_{i}(a a) \equiv 0,
$$

the hypercomplex consists of circles in involution with a given circle and the axial cross has disappeared.

Two proper crosses will have a complex invariant which we may write

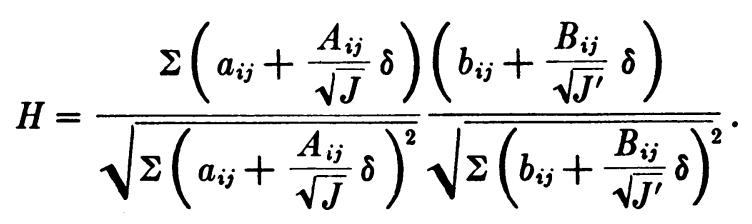

We find the interpretation thereof in terms of the circles of the crosses as follows. We may replace our hypercomplexes by such other hypercomplexes 
with the same axial crosses that these first invariants $I$ and $I^{\prime}$ are zero.

$$
\begin{gathered}
\alpha_{i j}=i a_{i j}+\frac{A_{i j}}{\sqrt{J},} \quad \alpha_{i j}^{\prime}=i a_{i j}-\frac{A_{i j}}{\sqrt{J}}, \\
\beta_{i j}=i b_{i j}+\frac{B_{i j}}{\sqrt{J^{\prime}}}, \quad \beta_{i j}^{\prime}=i b_{i j}-\frac{B_{i j}}{\sqrt{J^{\prime}}}, \\
\Sigma \alpha_{i j}^{2}=2 i \sqrt{J}, \quad \Sigma \alpha_{i j}^{\prime 2}=-2 i \sqrt{J} \\
\Sigma \beta_{i j}^{2}=2 i \sqrt{J^{\prime}}, \quad \Sigma \beta_{i j}^{\prime 2}=-2 i \sqrt{J^{\prime}}, \\
I(\alpha \beta)-I\left(\alpha^{\prime} \beta^{\prime}\right)=\frac{\Sigma\left[a_{i j} \frac{B_{i j}}{\sqrt{J^{\prime}}}+b_{i j} \frac{A_{i j}}{\sqrt{J}}\right]}{\sqrt[4]{J} \sqrt[4]{J^{\prime}}}, \\
I\left(\alpha \beta^{\prime}\right)-I\left(\alpha^{\prime} \beta\right)=\frac{\Sigma\left[a_{i j} b_{i j}+\frac{A_{i j}}{\sqrt{J}} \frac{B_{i j}}{\sqrt{J^{\prime}}}\right]}{\sqrt[4]{J} \sqrt[4]{J^{\prime}}}, \\
H=\frac{1}{2}\left\{\left[I(\alpha \beta)-I\left(\alpha^{\prime} \beta^{\prime}\right)\right]+\left[I\left(\alpha \beta^{\prime}\right)-I\left(\alpha^{\prime} \beta\right)\right] \delta\right\} .
\end{gathered}
$$

The strict truth is that we may so choose between the two circles of each cross that this last equation shall be fulfilled.

We pass now to a discussion of the axial crosses of the simplest one and two parameter families of linear hypercomplexes. The following theorem becomes evident when we remember that the central sphere of a linear hypercomplex is $x_{4}=0$ when, and only when $a_{i 4} \equiv 0$.

THEOREM 26: If two general linear hypercomplexes have the same central sphere, then this is the central sphere of every linear hypercomplex of the pencil linearly dependent on them. The hypercomplexes of the pencil will either all have the same axial crosses, or else these crosses will generate a cyclide or pseudocylindroid.

The general case of a pencil of linear hypercomplexes is incredibly harder to handle. Let us write

$$
\begin{gathered}
c_{i j}=y a_{i j}+b_{i j}, \\
\Omega_{i}(a a) \neq p \Omega_{i}(b b), \\
\Omega_{i}(c c)=y^{2} \Omega_{i}(a a)+2 y \Omega_{i}(a b)+\Omega_{i}(b b) .
\end{gathered}
$$

The central spheres will all pass through two points while their centers (in euclidean space) will generate a conic. The spheres will thus generate a twohorn cyclide. We have the further equations

$$
\begin{gathered}
\Sigma c_{i j}^{2}=y^{2} I+2 y \Sigma a_{i j} p_{i j}+I^{\prime}, \\
\Sigma \Omega_{i}^{2}(c c)=y^{4} J+4 y^{3} \Sigma \Omega_{i}(a a) \Omega_{i}(a b)+2 y^{2}\left[2 \Sigma \Omega_{i}^{2}(a b)+\Sigma \Omega_{i}(a a) \Omega_{i}(b b)\right]
\end{gathered}
$$


If $(\gamma)$ be an axial circle

$$
\gamma_{i j}=x c_{i j}+C_{i j},
$$

$x$ must be the solution of an equation like (35), namely

$$
\begin{aligned}
& x^{2}+2 x\left[y^{2} I+2 y \Sigma a_{i j} b_{i j}+I^{\prime}\right]+y^{4} J+4 y^{3} \Sigma \Omega_{i}(a a) \Omega_{i}(a b) \\
& \quad+2 y^{2}\left[2 \Sigma \Omega_{i}(a b)^{2}+\Sigma \Omega_{i}(a a) \Omega_{i}(a b)\right]+4 y \Sigma \Omega_{i}(a b) \Omega_{i}(b b)+J^{\prime}=0 .
\end{aligned}
$$

Each axial circle of our series will correspond to a pair of values of $x$ and $y$ satisfying these equations, each such pair of values will give one of our circles. The two values of $x$ corresponding to the same value of $y$ will give the two circles of a cross. If

$$
\left(\Sigma a_{i j} b_{i j}\right)^{2} \neq \Sigma a_{i j}^{2} \Sigma b_{i j}^{2},
$$

which we may assume to be the case, we may take

$$
I=I^{\prime}=0 \text {, }
$$

for $I$ will vanish for two hypercomplexes of the pencil. Our equation then takes the form

$$
(x+l y)^{2}=f^{(4)}(y) .
$$

THEOREM 27: If two general linear hypercomplexes do not have the same central sphere the axial circles of a pencil of such hypercomplexes linearly dependent on them will generate, in general, an elliptic series of circles.

If $\gamma_{i j}$ belong to an arbitrary linear hypercomplex

$$
\Sigma s_{i j} \gamma_{i j}=0
$$

we have an equation of the type

$$
(a y+b) x+\phi^{(3)}(y)=0 .
$$

Eliminating $x$ we get a sextic in $y$; each $y$ will correspond to but one $x$.

TheOREM 28: Six circles of the series will belong to an arbitrary linear hypercomplex.

Let us find the order of the surface swept out by the circles. If $(z)$ be a point on one of our circles

$$
\sum_{n} \gamma_{i n} z_{n}=\sum_{n} \gamma_{j n} z_{n}=0
$$

These will yield two equations

$$
(a y+b) x+\varphi^{8}(y)=0, \quad\left(a^{\prime} y+b^{\prime}\right) x+\varphi^{(3)}(y)=0 .
$$

The elimination of $x$ and $y$ between these two and (38) is equivalent to eliminating $x y$ from

$$
(a y+b) x+\varphi^{3}(y)=\left(a^{\prime} y+b^{\prime}\right) x+{\varphi^{\prime}}^{(3)}(y)=x^{2}-\psi^{(4)}(y)=0 .
$$


These yield the equations

$$
(a y+b)^{2} \psi^{(4)}(y)=\left[\phi^{(3)}\right]^{2}, \quad(a y+b) \phi^{(3)}-\left(a^{\prime} y+b^{\prime}\right) \phi^{(3)}=0 .
$$

If these latter be satisfied, so are the original three, or else

$$
(a y+b)=\varphi^{(3)}=0 .
$$

The resultant of a quartic and a sextic in $(y)$ whose coefficients are quadratic in $(z)$ will be an equation of the twentieth degree in $(z)$. This will contain the resultant of a linear and a cubic equation, which is of the fourth degree in $(z)$, as a quadratic factor. We must therefore split off from our expression of the twentieth degree one of the eighth degree.

Theorem 29: If two linear hypercomplexes have different central spheres, the axial circles of the pencil of linear hypercomplexes linearly dependent on them will generate a surface of the twenty-fourth order.

We pass now to the general net of linear hypercomplexes, i. e., those linearly dependent on three whereof no two have the same central sphere. Here we have

$$
\begin{gathered}
d_{i j}=y a_{i j}+z b_{i j}+c_{i j}, \\
\delta_{i j}=x d_{i j}+D_{i j}, \\
x^{2}+2 x\left[y^{2} \Sigma a_{i j}^{2}+z^{2} \Sigma b_{i j}^{2}+2 y z \Sigma a_{i j} b_{i j}+2 y \Sigma a_{i j} c_{i j}+2 z \Sigma b_{i j} c_{i j}+\Sigma c_{i j}^{2}\right] \\
+\phi^{(4)}(y z)=0 .
\end{gathered}
$$

We may, in general, assume that

$$
\Sigma a_{i j}^{2}=\Sigma b_{i j}^{2}=\Sigma a_{i j} b_{i j}=0 .
$$

Our equation takes the reduced form, in homogeneous coördinates

$$
(x+l y+m z)^{2} t^{2}+f^{(4)}(y z t)=0 .
$$

This may be linearly transferred to

$$
x^{\prime 2} t^{2}-\left(a y^{\prime 2}+b y^{\prime} t^{\prime}+c t^{\prime 2}\right)^{2}+z^{\prime} f^{3}\left(y^{\prime} z^{\prime} t^{\prime}\right)=0 .
$$

The circles of our axial congruence are in one-to-one correspondence with the points of a quartic surface which has no singularity except the extremity of one axis. The really significant feature of this surface is that it is rational and can be carried over into a cubic surface by a quadratic Cremona transformation.*

$$
\begin{aligned}
\rho X & =x^{\prime} t^{\prime}+a y^{\prime 2}+b y^{\prime} t^{\prime}+c t^{2}, & \sigma x^{\prime} & =X Z-\left(a Y^{2}+b Y T+c T^{2}\right), \\
\rho Y & =y^{\prime} z^{\prime}, & \sigma y^{\prime} & =Y T, \\
\rho Z & =z^{\prime 2}, & \sigma z^{\prime} & =Z T, \\
\rho T & =z^{\prime} t^{\prime}, & \sigma t^{\prime} & =T^{2} .
\end{aligned}
$$

*C. Cremona: Sopra un certo superficie di quart'ordine, Collectanea Matematica, Naples, 1881. 
Here it it must be remembered that $y^{\prime} z^{\prime} t^{\prime}$ are linear and homogeneous in our original $y z t$. The cubic surface may be expressed parametrically by putting $X Y Z T$ as functions quadratic in each of two independent variables $u v$. Hence for our quartic

$$
x=f^{4}(u v), \quad y=\phi f_{1}^{(2)}(u v), \quad z=\phi f_{2}^{(2)}(u v), \quad f=\phi f_{3}^{(2)}(u v) .
$$

Now $d_{i j}$ is linear and homogeneous in $y z t$, and the common factor $\phi$ may be struck therefrom, we thus get for the circles of our congruence the parametric expressions

$$
\delta_{i j}=f^{4}(u v) f_{j}^{(2)}(u v)+\phi_{i j}^{(6)}(u v) .
$$

It must be remembered that here the superscript indicates the degree in $u$ and $v$ separately, not the total degree.

Theorem 32: If no two linear hypercomplexes of a net have the same central sphere the coördinates of their axial circles may be expressed as rational functions of two independent variables each entering to the sixth order; seventy-two of these circles will belong to two arbitrary linear hypercomplexes.

We find the focal surface of the congruence as follows. A sphere $(z)$ will be orthogonal to a circle $(\delta)$ if

$$
\sum_{n} \delta_{i n} z=\sum \delta_{j n} z_{n}=0 \quad \delta_{i j} \neq 0 .
$$

We may for an arbitrary ( $z$ ) surely choose $i$ and $j$ so that these three conditions are satisfied. The equation which we desire is obtained by equating to zero the tact-invariant of these looked upon as two curves in the $u v$ plane. The order of this in the coefficients of one of the curves is the number of free intersections of this curve with the Jacobian of the two given curves and a third of the same structure.* Each of our curves is of the twelfth order with a sextuple point at the end of each axis. The Jacobian is of the thirty-third order, but contains $u$ and $v$ only to the seventeenth order; it has therefore a point of the sixteenth order at the extremity of each axis. The Jacobian intersects each curve three hundred ninety-six times, but one hundred ninety-two intersections are at the ends of the axes; there remain two hundred four free intersections; this is therefore the order of the tact invariant in the coefficients of each curve, or one half of its order in $(z)$.

Theorem 33: The axial circles of a general net of linear hypercomplexes envelop a surface of the order eight hundred sixteen.

There is more satisfaction in finding the order of such a surface than in seeking its equation.

The general cubic surface contains twenty-seven straight lines, and these transform into parabolas of the quartic surface. The plane of each of these parabolas will meet the quartic in a second parabola with a parallel axis. There was one parabola common to all the homaloidal paraboloids of the

\footnotetext{
- Cf. Samon, Higher Plane Curves, third ed., p. 81.
} 
Cremona transformation, and another in its plane. Beyond these fifty-six there are no parabolas on the quartic.* Each of these may be expressed in the form

$$
\begin{gathered}
x=\phi^{(2)}(s), \quad y=(a s+b)\left(a_{1} s_{1}+b_{1}\right), \quad z=(a s+b)\left(a_{2} s+b_{2}\right), \\
t=(a s+b)\left(a_{3} s+b_{3}\right) .
\end{gathered}
$$

We see that $d_{i j}$ is linear, and $\delta_{i j}$ cubic in $s$.

ThEOREM 34: The congruence contains fifty-six surfaces of the twelfth order.

\section{§4. An Involutory Cirale Transformation.}

If two non-cospherical circles $(p)$ and $(q)$ be given, there will be just one circle orthogonal to their common orthogonal sphere, and in bi-involution with $(p)$. The coördinates of this sphere are $\Omega_{i}(p q)$; we wish for the circle orthogonal to this and to the two null spheres $(x)$ and $(y)$ whose vertices are the foci of $(p)$. The circle required will thus, by (23), have the Plücker coördinates

$$
\begin{gathered}
P_{i j}=\left|\begin{array}{lll}
x_{k} & x_{l} & x_{m} \\
y_{k} & y_{l} & y_{m} \\
\Omega_{k} & \Omega_{l} & \Omega_{m}
\end{array}\right|, \\
P_{i j}=p_{l m} \Omega_{k}(p q)+p_{m k} \Omega_{l}(p q)+p_{k l} \Omega_{m}(p q) .
\end{gathered}
$$

If a fundamental circle $(q)$ be given, then each circle $(p)$ not cospherical with it will correspond in an involutory way to a circle $(P)$, the relation between the two being that they are in bi-involution, and their common orthogonal sphere is orthogonal to $(q)$. Another way of stating this is to say that $(P)$ is the circle through the foci of $(p)$ orthogonal to the sphere through those foci and the foci of the fundamental circle. The relation of $(p)$ to $(P)$ is involutory. The most striking feature about this transformation, however, is that it does not carry cospherical circles into cospherical circles. $\dagger$ We find in fact

$$
\Omega_{m}\left(P P^{\prime}\right)=\left|\begin{array}{cccccc}
0 & x_{i} & x_{j} & x_{k} & x_{l} & x_{m} \\
0 & y_{i} & y_{j} & y_{k} & y_{l} & y_{m} \\
x_{m}^{\prime} & x_{i}^{\prime} & x_{j}^{\prime} & x_{k}^{\prime} & x_{i}^{\prime} & 0 \\
y_{m}^{\prime} & y_{i}^{\prime} & y_{j}^{\prime} & y_{k}^{\prime} & y_{i}^{\prime} & 0 \\
0 & \Omega_{i}(p q) & \Omega_{j}(p q) & \Omega_{k}(p q) & \Omega_{l}(p q) & \Omega_{m}(p q) \\
\Omega_{m}\left(p^{\prime} q\right) & \Omega_{i}\left(p^{\prime} q\right) & \Omega_{j}\left(p^{\prime} q\right) & \Omega_{k}\left(p^{\prime} q\right) & \Omega_{l}\left(p^{\prime} q\right) & 0
\end{array}\right|
$$

* Cremona, loc. cit., p. 418.

$\dagger$ Previous writers do not seem to have noticed circle transformations which were not also sphere transformations. It is the more remarkable that the first of these to be studied should be involutory. 
Assuming that $(p)$ and $\left(p^{\prime}\right)$ are cospherical so that $\Omega_{i}\left(p p^{\prime}\right) \equiv 0$, we have

$$
\begin{aligned}
\Omega_{m}\left(P P^{\prime}\right)=\Sigma\left[\Omega_{l}(p q) \Omega_{m}\left(p^{\prime} q\right)-\Omega_{m}(p q) \Omega_{l}\left(p^{\prime} q\right)\right] & \\
& {\left[p_{j k} p_{m i}^{\prime}+p_{k i} p_{m j}^{\prime}+p_{i j} p_{m k}^{\prime}\right] } \\
& +\Sigma\left[\Omega_{j}(p q) \Omega_{k}\left(p^{\prime} q\right)-\Omega_{k}(p q) \Omega_{j}\left(p^{\prime} q\right)\right]\left[p_{m i}^{\prime} p_{l m}-p_{m i} p_{l m}^{\prime}\right] .
\end{aligned}
$$

This will vanish for all values of $m$ and for all pairs of cospherical circles when

$$
\Omega_{i}(p q) \equiv \Omega_{i}\left(p^{\prime} q\right) .
$$

TheOREM 35: The transformation which carries each circle not cospherical with a fundamental circle into the circle in bi-involution with it, and orthogonal to the sphere which is orthogonal to it and to the fundamental circle, is single valued and involutory, but will in general carry cospherical circles into cospherical circles when, and only when, the common sphere is orthogonal to the fundamental circle.

It will be found also that the properties of involution and bi-involution are not invariant under the transformation, neither is it usually a contact transformation.

Suppose that $(p)$ traces a pencil of circles including one that is cospherical with $\left(q_{j}\right)$; we shall have

$$
P_{i j}=\lambda A_{i j}+\mu B_{i j} .
$$

When no member of the pencil is cospherical with $(q)$ we have

$$
P_{i j}=\lambda^{2} R_{i j}+2 \lambda \mu A_{i j}+\mu^{2} S_{i j} \text {. }
$$

All of these circles are orthogonal to the sphere generated by the pencil. There are but three conditions imposed upon a circle orthogonal to this sphere to require it to be cospherical with all of the circles of this system.

TheOREM 36: A pencil of circles containing one member cospherical with the fundamental circle is transformed into another such pencil; a pencil of circles containing no such member is transformed into one generation of a cyclide anallagmatic in the sphere generated by the given pencil.

It would be pleasant if the circles of the pencil cospherical and orthogonal to the first transformed into the conjugate generation of the same cyclide; • such is not, however, the case. We find the transforms of the circles of a sphere which does not contain $(q)$ as follows. Let us take as the sphere $x_{4}=0$, which we may assume has no special relation to $(q) ;(p)$ will have only those coördinates which have a subscript $4, P$ will lack all coördinates with this subscript, so that $(P)$ is orthogonal to that sphere. We find also, in the notation of section 2 , that $(P / q)=0$.

Theorem 37: The circles of a sphere not containing the fundamental circle are transformed into the circles orthogonal to that same sphere and cospherical with the circle which meets it orthogonally in the same points as the fundamental circle. 
This theorem will hold by continuity even when the sphere is null. If the circles on the given sphere be orthogonal to a fixed circle one more linear condition is imposed on $(P)$. If two conditions of this type be imposed upon $(P)$ we have, by (36), one generation of a cyclide, hence

THEOREM 38: The circles of a sphere which does not contain the fundamental circle which cut orthogonally a fixed circle of that sphere will be transformed into circles cospherical with two fixed circles orthogonal to that sphere.

Suppose that we have a system of circles through two points. Their foci trace the circle whose foci are these two points, and any two points of this circle are foci of a circle through the original two points.

Theorem 39: The circles through two fixed points, not on the fundamental circle, are transformed into the circles through each pair of points of the circle whose foci are the fixed points, orthogonal in each case to the sphere connecting the two required points with the foci of the fundamental circle.

THEOREM 40: The circles orthogonal to a sphere which does not contain the fundamental circle are transformed into the circles through pairs of points of this sphere, orthogonal in each case to the sphere connecting the pairs of points with the foci of the fundamental circle.

Let us find the transforms of all circles which are cospherical with a given circle $(r)$. Since $(p)$ and $(r)$ are cospherical, their foci are concyclic; $(p)$ transforms into the circle through its foci, orthogonal to the sphere containing them and the foci of $(q)$. If we take an arbitrary sphere through the foci of $(q)$, the points thereon concyclic with the foci of $(r)$ are corresponding points in the inversion whose fundamental sphere is the sphere through $(r)$ orthogonal to the sphere in question.

THEOREM 41: The circles cospherical with a given circle not cospherical nor in bi-involution with the fundamental circle are the circles orthogonal to each sphere through the foci of the fundamental circle at pairs of points which are mutually inverse in the sphere through the given circle and orthogonal to the sphere in question.

When $(q)$ and $(r)$ are cospherical, every circle through the foci of $(r)$ lies on a sphere through the foci of $(q)$.

ThEOREM 42: The circles cospherical with a circle which is cospherical with the fundamental circle transform into the circles in involution with the given circle and with the circle containing the foci of the given and the fundamental circle.

Harvard University,

Cambridge, Mass., June, 1912. 